\title{
Characterization of a ferroelectric $\mathrm{BaTiO}_{3} / \mathrm{SrTiO}_{3}$ heterostructure with interface- induced polarization
}

\author{
HsinWei Wu, ${ }^{1}$ Toshihiro Aoki, ${ }^{2}$ Patrick Ponath, ${ }^{3}$ Alexander A. Demkov, ${ }^{3}$ Martha R. McCartney, ${ }^{4}$ \\ and David J. Smith ${ }^{4}$ \\ ${ }^{1 .}$ School of Engineering for Matter, Transport, and Energy, Arizona State University, Tempe, AZ 85287 \\ 2. LeRoy Eyring Center for Solid State Science, Arizona State University, Tempe, AZ, 85287 \\ 3. Department of Physics, The University of Texas at Austin, Austin, TX 78712 \\ 4. Department of Physics, Arizona State University, Tempe, AZ, 85287
}

$\mathrm{BaTiO}_{3}$ (BTO) is a promising candidate for future applications in ferroelectric field-effect transistors because of its high permittivity and low-temperature ferroelectricity. For transistor devices, out-of-plane polarization of BTO, i.e., $c$-axis of BTO tetragonal structure along the growth direction, is preferred. In this work, BTO/STO heterostructures were deposited with strained interfaces in order to control the phase and polarization orientation. 15-20 nm thin films of single-crystal BTO were grown by molecular beam epitaxy (MBE) on either STO substrates or on 2-nm-thick STO buffer layers with $\mathrm{Ge}(001)$ substrates. When BTO is directly deposited on Ge substrates, there is a $45^{\circ}$ in-plane rotation, and the reduced lattice parameter of Ge, $4.001 \AA$, is slightly larger than that of BTO, $3.994 \AA$. The BTO film is then under tensile strain and in-plane polarized [1]. Conversely, when BTO is deposited on $\mathrm{SrTiO}_{3}(\mathrm{STO})$ substrate or buffer layer (lattice constant 3.905 $\AA$ ), it is compressively strained and shows out-of-plane polarization [2]. Samples for TEM observation were prepared via standard cross-section method with argon-ion-milling. Aberration-corrected STEM images were recorded using a JEOL ARM 200F operated at $200 \mathrm{keV}$.

Figures 1(a) to (f) are HAADF and BF STEM images for BTO layer deposited on STO buffer layer and Ge substrate. Figures 1(g) and (h) are HAADF and BF STEM images for BTO deposited directly on STO substrate. Figures 1(a) and (b) show a sharp STO/Ge interface with $2 \times 1$ surface reconstruction, and in figs. 1(c) and (d), steps between $2 \times$ and $1 \times$ periodicities on the Ge substrate surface can be clearly seen. The STO unit cells are under tensile strain and elongated horizontally because of lattice mismatch between STO/Ge. In BTO/STO/Ge samples, no misfit dislocations were observed; instead, discontinuous structural changes, shown in figs. 1(e) and (f), are visible, which are believed to be caused by anti-phase boundaries. On the contrary, for samples with BTO directly deposited on STO substrate, both misfit dislocations and anti-phase boundaries were observed, as visible in figs. 1(g) and (h). BTO layers in both BTO/STO/Ge and BTO/STO heterostructures retain $c$-axis-oriented tetragonal structure. However, atomic displacements with different interface structures are measured, and BTO grown on STO substrate shows stronger Ti shift compared to BTO/STO/Ge heterostructures. Ferroelectric domain boundaries and any possible relationship with dislocations are being investigated by electron holography.

References:

[1] K. D. Fredrickson, et al., Appl. Phys. Lett. 104 (2014) 242908.

[2] P. Ponath, et al., Nat. Commun. 6 (2015) 6067.

[3] This work was supported by AFOSR Contract FA 9550-12-10494. We gratefully acknowledge the use of facilities within the John M. Cowley Center for High Resolution Electron Microscopy at Arizona State University. 

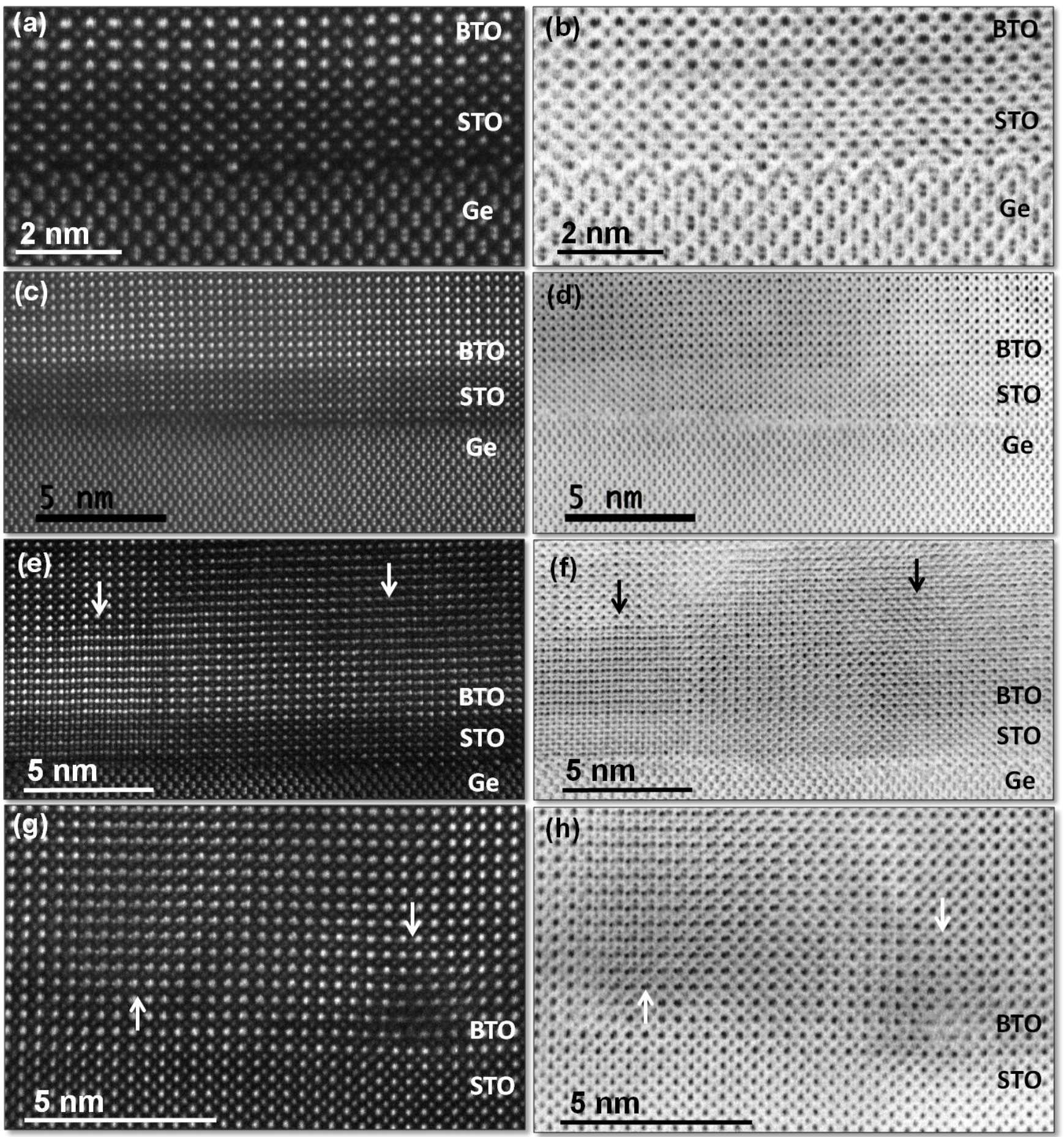

Fig. 1. (a) $\mathrm{HAADF}$, and (b) BF, images of BTO/STO/Ge showing sharp STO/Ge interface with $2 \times 1$ Ge surface reconstruction. (c) HAADF, and (d) BF, images of BTO/STO/Ge showing Ge surface steps between $2 \times$ and $1 \times$ periodicities. (e) $\mathrm{HAADF}$, and (f) $\mathrm{BF}$, images of $\mathrm{BTO} / \mathrm{STO} / \mathrm{Ge}$ showing large discontinuous contrast difference from boundaries. (g) HAADF, and (h) BF, images of BTO/STO showing both discontinuous contrast difference (left arrow) and a misfit dislocation (right arrow). 